\title{
Protective effects of fasudil hydrochloride post-conditioning on acute myocardial ischemia/reperfusion injury in rats
}

\author{
Zhi-hui Jiang, Tian-tian Zhang, Jun-feng Zhang \\ Department of Cardiology, No. 3 People's Hospital affiliated \\ to Shanghai Jiao Tong University School of Medicine, Shanghai, China
}

\begin{abstract}
Background: In recent years, the alleviation of acute myocardial ischemia and reperfusion injury (MI/RI) during myocardial reperfusion has presented a significant clinical challenge. This study was performed to investigate the effects of fasudil hydrochloride (FH) postconditioning on MI/RI and the underlying mechanism.
\end{abstract}

Methods: Seventy-two rats were randomly divided into four groups: a Sham group, an ischemia/reperfusion (I/R) group, a fasudil hydrochloride (FH) group, and a fasudil hydrochloride + PI3K inhibitor $(F H+I)$ group. Myocardial infarct size, cell apoptotic index (AI), and myocardial tissue expression of Rho-associated coiled-coil containing protein kinase 1 (ROCK1), Bcl-2, Bcl-2 associated X protein (Bax), caspase-3, Akt and phosphorylated Akt (P-Akt) were detected.

Results: All these parameters, except Akt expression, were higher in the I/R group than in the Sham group ( $p<0.05)$. Compared to the I/R group, myocardial infarct size, AI, Bax and caspase-3 expression were significantly reduced in the $\mathrm{FH}$ group $(p<0.05)$, while Bcl-2 expression was increased $(p<0.05)$. However, the myocardial infarct size and AI of the $F H+I$ group were similar to those of the I/R group ( $p>0.05$ ). Compared to the FH group, Bcl-2 expression was reduced in the FH+I group, while Bax and caspase-3 expression was increased $(p<0.05)$. Furthermore, $P$-Akt expression in the FH group was significantly higher than that of the $I / R$ group $(p<0.05)$.

Conclusions: FH post-conditioning alleviated MI/RI, with narrowing of the infarct size and decreased apoptosis of ischemic cardiocytes. The mechanism was associated with activation of the PI3K-Akt signaling pathway. (Cardiol J 2013; 20, 2: 197-202)

Key words: fasudil hydrochloride (FH), ischemia/reperfusion, post-conditioning

\section{Introduction}

Acute myocardial infarction (AMI) is a common acute disease in cardiology with a high mortality rate $[1,2]$. Clearing obstructed vessels rapidly and effectively is critical for the treatment of AMI [3].
However, reperfusion is closely associated with metabolic dysfunction and structural damage to the cardiocytes, which is known as myocardial ischemia/reperfusion injury (MI/RI) [4]. Therefore, alleviating $\mathrm{MI} / \mathrm{RI}$ during myocardial reperfusion represents a significant clinical challenge. Re-

Address for correspondence: Jun-feng Zhang, MD, Department of Cardiology, No. 3 People's Hospital affiliated to Shanghai Jiao Tong University School of Medicine, No. 280, Mohe Road, Baoshan District, 201900 Shanghai, China, tel: +8621566 911 01-6260, fax: +86 21566916 62, e-mail: zhangjf1222@yahoo.com.cn

Jiang Zhi-hui and Zhang Tian-tian contributed equally to this article on the conception, design, execution and interpretation of the data, and the written of the paper. Jiang Zhi-hui is now working in Department of Cardiology, Taixing People's Hospital, Jiangsu, China.

Received: 23.08 .2012

Accepted: 15.11 .2012 
searchers [5] have proposed ischemic pre-conditioning (IPC) to address this issue. But the application value of IPC is rare in AMI patients. Ischemic post-conditioning (IPost) was first demonstrated in 2003 in a study of ischemia/reperfusion in dogs [6] and its clinical value in protecting the myocardium and improving prognosis was subsequently verified $[7,8]$. However, the application of IPost is also limited as it is time-consuming and carries numerous serious complications. In recent years, a variety of agents such as adenosine, bradykinin (BK) and erythropoietin have been shown to activate the same signaling pathways that IPC and IPost mediate, mitigating MI/RI $[9,10]$. This is known as pharmacological post-conditioning (PPost) [11]. PPost has become a significant focus of clinical research, with encouraging application prospects for its convenience and effectiveness.

The mechanism underlying MI/RI includes oxygen free radical injury, calcium overload, endothelial dysfunction and cell apoptosis, which is an important contributing factor. Decreased Bcl-2 and increased $\mathrm{Bcl}-2$ associated $\mathrm{X}$ protein (Bax) induces opening of the mitochondrial permeability transition pore (mPTP), resulting in the release of effectors such as cytochrome $\mathrm{c}$ from mitochondria, initiating the caspase cascade, leading to cell apoptosis [12$-15]$. However, reperfusion injury salvage kinase (RISK) inhibits apoptosis by blocking the process [15-17]. During early reperfusion, Rho associated coiled-coil forming protein kinase (ROCK) was activated, suppressing the RISK pathway [18, 19].

Fasudil hydrochloride $(\mathrm{FH})$, which is the first specific ROCK inhibitor that has been applied in clinical practice, functions by competing with ATP for the ATP binding site in the Rho kinase catalytic domain [20]. Ichinomiya et al. [21] have shown that high-dose $\mathrm{FH}$ preserves post-conditioning against myocardial infarction via the activation of m-KATP channels [21]. However, many other specific mechanisms about $\mathrm{FH}$ postconditioning are still unclear. So the discovery of more details, for example the relationship between $\mathrm{FH}$ and ROCK expression, $\mathrm{FH}$ and apoptosis, would be valuable. Previously, intravenous injection was the most commonly adopted route of administration, while intracoronary injection was rare. Researchers [22] have hypothesized that intracoronary injection might improve the efficiency of PPost, based on the observation that intracoronary administration of edaravone was more effective in the protection of the myocardium during MI/RI. Increasing experience of percutaneous interventions in clinical practice makes intracoronary injection possible. Furthermore, it is thought that direct application of the drug to the target organ will limit the reduction in the drug concentration due to distribution to various organs, thus greatly improving the utilization rate of the drug.

In this study, an in vivo myocardial ischemia and reperfusion model was established in rats by ligating the coronary artery with a balloon. Intracoronary injection was adopted to test the effectiveness of $\mathrm{FH}$ post-conditioning and to explore the possible apoptosis mechanism.

\section{Methods}

Animals in this work were treated in accordance with all guiding principles in the care and use of animals. All procedures were approved by the local ethical committee.

\section{Reagents}

Fasudil hydrochloride (HA1077, Chuan Wei, lotnumber 1011051, Tianjin Chase Sun Pharmaceutical Corporation, China), nitrotetrazolium blue chloride (NBT, Shanghai Haoran Biotechnologies Corporation, Ltd., China), Rho-associated coiled-coil containing protein kinase 1 (ROCK1) antibody, Bcl-2 antibody, Bax antibody and caspase- 3 antibody (Abcam Corporation, Ltd., UK), Akt and phosphorylated Akt (P-Akt) antibodies (Cell Signal Corporation, Ltd., US).

\section{Myocardial ischemia and reperfusion model}

Healthy and specific pathogen free male Sprague Dawley (SD) rats $(\mathrm{n}=72,250 \pm 10 \mathrm{~g})$ were selected. The animals were provided by the Experimental Animal Center of the Second Military Medical University and maintained in 12 cages (six per cage) at $23 \pm 2{ }^{\circ} \mathrm{C}$, and $60-65 \%$ relative humidity and given free access to food and water.

The in vivo myocardial ischemia and reperfusion model was established in rats by the ligating the coronary artery with a balloon. Animals were anesthetized and respiration was assisted with a ventilator. A polyethylene plastic catheter $(0.8 \mathrm{~mm}$ internal diameter) connected to the Power Lab physiological experimental system was inserted into the right common carotid artery until a characteristic left ventricular pressure wave was observed. The chest was opened via the fourth or fifth left lateral thoracotomy. The pericardium was opened and the heart was exposed. A high-pressure balloon was placed on the left anterior descending artery (LAD). The myocardial ischemia was performed by ligating the LAD with the balloon $(3.0 \times$ $\times 10 \mathrm{~mm}, 12 \mathrm{~atm}$ ) with a $6-0$ silk suture around the 
LAD at $1 \mathrm{~mm}$ distal to the left atrial appendage. The reperfusion was performed by emptying the balloon $(0 \mathrm{~atm})$ to re-open the LAD.

$\mathrm{SD}$ rats $(\mathrm{n}=72)$ were randomly divided into four groups (18 per group) as follows: (1) The Sham group: the LAD was encircled by a 6-0 silk suture without ligation for $180 \mathrm{~min}$; (2) Ischemia/reperfusion (I/R) group: after ligation for $60 \mathrm{~min}$, the pressure was adjusted to $0 \mathrm{~atm}$ to restore blood flow for 120 min; (3) Fasudil hydrochloride (FH) group: after ligation for $60 \mathrm{~min}$, the pressure pump was adjusted to $0 \mathrm{~atm}$. A micro-injection pump was connected to the polyethylene plastic catheter in the right common carotid artery. The catheter was pulled from the left ventricle to the aortic root, where the left ventricular pressure wave disappeared. FH (500 $\mu \mathrm{g} /$ $/ \mathrm{mL}$, dissolved in normal saline) was pumped into the aortic root $(500 \mu \mathrm{g} / \mathrm{kg} / \mathrm{min} \times 5 \mathrm{~min})$, followed by reperfusion for $115 \mathrm{~min}$; (4) Fasudil hydrochloride + PI3K inhibitor $(\mathrm{FH}+\mathrm{I})$ group: at $10 \mathrm{~min}$ before ligation, the PI3K inhibitor LY-294002 $(75 \mu \mathrm{g} /$ $/ \mathrm{mL}$, dissolved in normal saline) was administered (dose, $1 \mathrm{~mL} / \mathrm{kg}$ ). Subsequently, animals received identical treatment to the $\mathrm{FH}$ group.

\section{Immunohistochemical analysis}

At the end of reperfusion, six SD rats randomly selected from each group were sacrificed by cervical vertebra dislocation. Hearts were removed and washed with normal saline. After being dried with filter paper, the total ventricle was weighed. The ventricle was cut into five slices of equal thickness from the cardiac apex to the cardiac base, in parallel with the atrioventricular groove. Tissue slices were stained with $0.1 \% \mathrm{NBT}$ at $37^{\circ} \mathrm{C}(10-20 \mathrm{~min})$ and excess dye was washed off with normal saline. Infarcted myocardium was not stained, while noninfarcted myocardium was stained black. After acquisition of digital photographs, the stained myocardium was removed and the unstained myocardium was weighed. The weight ratio of the infarcted myocardium to the total ventricle was calculated to determine the myocardial infarct size.

\section{Terminal deoxy-nucleotidyl transferase- -mediated dUTP-biotin nick end-labeling (TUNEL) assay}

Another six SD rats were randomly selected from each group. Myocardial blocks from anterior wall of left ventricle were cut off, fixed with neutral formalin for 24 hours and embedded in paraffin. Apoptotic cells were detected using terminal deoxy-nucleotidyl transferase-mediated dUTP nick end-labeling (TUNEL) assay according to the in- structions provided by the manufacturer. Normal myocardial nuclei were stained blue, while apoptotic nuclei were brown as visualized by general microscopy $(400 \times$ magnification). Five fields were randomly selected in each section for enumeration of TUNEL-positive myocytes and total myocytes. The percentage (\%) of TUNEL-positive myocytes was calculated to represent the apoptotic index.

\section{Western blot analysis}

A further six SD rats in each group were randomly selected. Hearts were removed. Total cellular protein isolated from the myocardium was separated by SDS-PAGE, and transferred to a nitrocellulose membrane. Target bands were detected by immunoblotting with various primary antibodies (ROCK1, Bcl-2, Bax, caspase-3, Akt, P-Akt). The membranes were probed with horseradish peroxidase conjugated secondary antibodies. Immunoreactive bands were visualized by enhanced chemiluminescence and quantified by densitometry.

\section{Calculations and statistics}

Data is presented as the mean \pm SD from at least three independent experiments. Statistical analysis was performed with Student's t-test by SPSS 17.0 software. A value of $p<0.05$ was considered statistically significant.

\section{Results}

1. FH reduced the myocardial infarct size in the acute myocardial ischemia-reperfusion model (Fig. 1). In the Sham group, the myocardial infarct size was 0 . The myocardial infarct sizes in the $\mathrm{FH}$ group $(8.62 \% \pm 3.04 \%)$ were reduced significantly compared to those of the I/R group $(18.25 \% \pm 5.19 \% ; \mathrm{p}<0.05)$. There was no difference in myocardial infarct sizes between the I/R group and the $\mathrm{FH}+\mathrm{I}$ group $(16.70 \% \pm 5.80 \% ; p>0.05)$.

2. FH decreased apoptosis in cardiocytes following reperfusion (Fig. 2). Few apoptotic cells $(7.11 \% \pm 1.24 \%)$ were detected in the Sham group. However, the number of apoptotic cells significantly increased in the other three groups $(\mathrm{p}<0.05)$. Compared to the $\mathrm{I} / \mathrm{R}$ group $(41.12 \% \pm 5.47 \%)$, significantly fewer apoptotic cells were detected in the $\mathrm{FH}$ group $(28.56 \% \pm$ $\pm 2.87 \%$; $<<0.05$ ), although there was no significant difference between the I/R group and the $\mathrm{FH}+\mathrm{I}$ group $(40.40 \% \pm 7.89 \%$; $\mathrm{p}>0.05)$.

3. ROCK1 expression in myocardial tissues was not influenced by FH (Fig. 3A). Compared to 


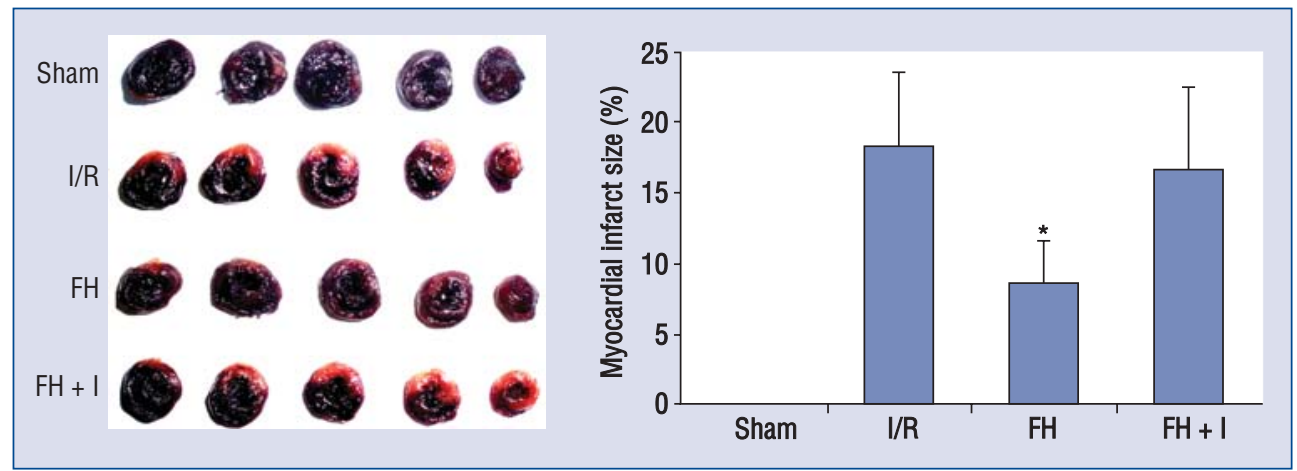

Figure 1. Myocardial infarct size in the acute myocardial ischemia-reperfusion model in rats in each group. FH narrowed the myocardial infarct size. Compared to I/R group; ${ }^{*} p<0.05$.

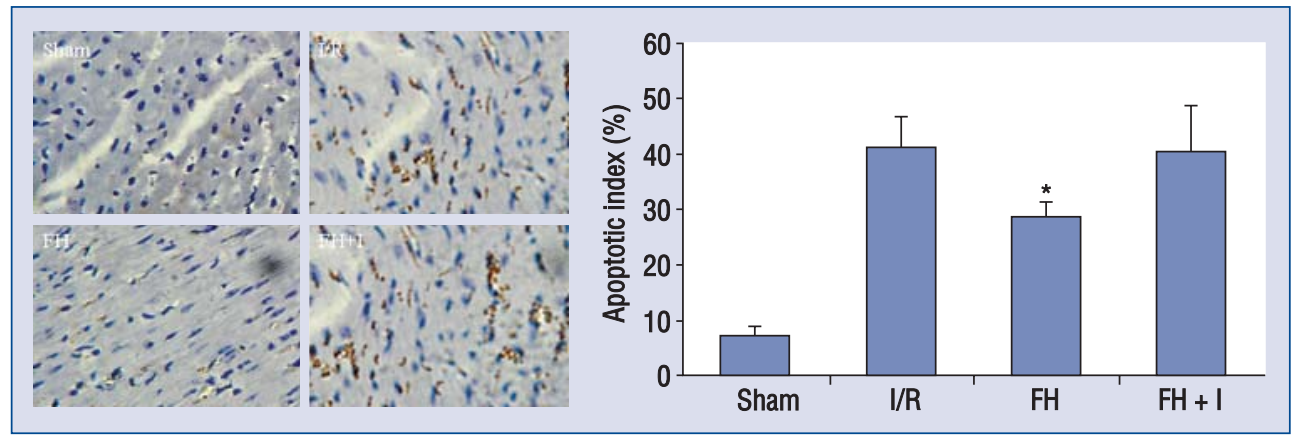

Figure 2. Apoptotic index of cardiocytes in each group. FH decreased apoptosis in ischemic cardiocytes. Compared to I/R group; ${ }^{*} p<0.05$.

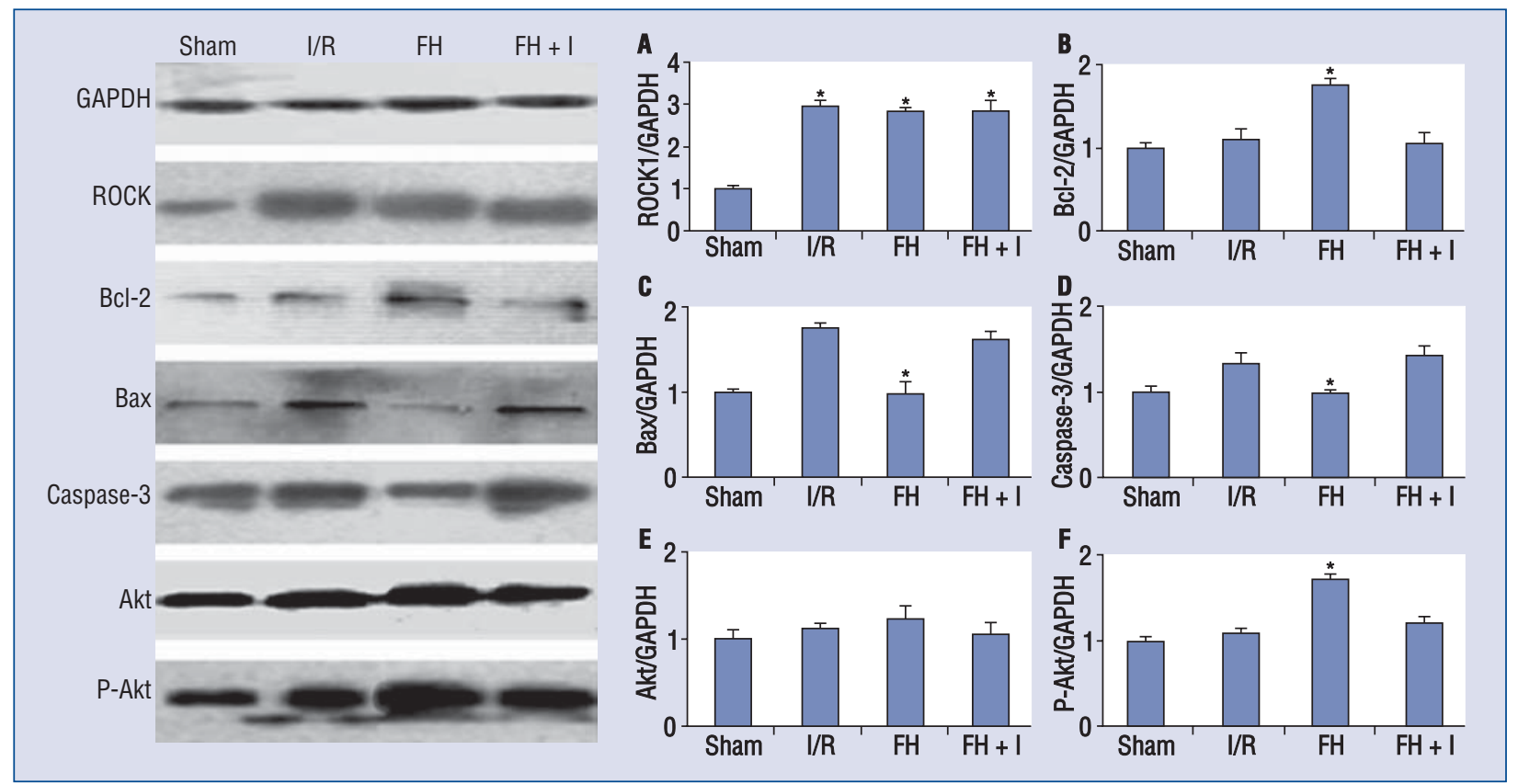

Figure 3. Expression of ROCK1, Bcl-2, Bax, Caspase-3, Akt and P-Akt in myocardial tissues in each group by Western blot. Compared to the Sham group, ROCK1 expression was increased by $\mathrm{FH}_{;}{ }^{*} \mathrm{p}<0.05$ (A). Compared to the I/R group, $\mathrm{FH}$ increased the expression of $\mathrm{Bcl}-2$ and $\mathrm{P}$-Akt in the acute myocardial ischemia reperfusion model (B, F), while it decreased the expression of Bax and Caspase-3 (C, D), * $p<0.05$. Compared to the I/R group, Akt expressions were not influenced by $\mathrm{FH},{ }^{*} \mathrm{p}>0.05$ (E) 
the Sham group, the ROCK1 protein expression in the other groups increased significantly ( $<<0.05)$, although there was no statistically significant difference between these groups $(\mathrm{p}>0.05)$.

4. FH increased the expression of Bcl-2 in the acute myocardial ischemia-reperfusion model (Fig. 3B). There was no significant difference in Bcl-2 expression between the Sham group and the I/R group (1.11 $\pm 0.12 ; p>0.05)$. Compared to the I/R group, Bcl-2 expression was increased in the FH group $(1.76 \pm 0.08 ; \mathrm{p}<$ $<0.05)$. Furthermore, no difference was detected between the I/R group and the $\mathrm{FH}+\mathrm{I}$ group (1.05 $\pm 0.12 ; \mathrm{p}>0.05)$.

5. FH decreased Bax expression in the acute myocardial ischemia-reperfusion model (Fig. 3C). Compared to the Sham group, Bax expression was significantly increased in the I/R group (1.75 $\pm 0.06 ; p<0.05)$. Compared to the I/R group, Bax expression was decreased in the FH group $(0.98 \pm 0.14 ; \mathrm{p}<0.05)$. There was no difference between the I/R group and the $\mathrm{FH}+\mathrm{I}(1.61 \pm 0.11)$ group $(\mathrm{p}>0.05)$.

6. $\mathrm{FH}$ decreased the expression of caspase- 3 in the acute myocardial ischemia-reperfusion model (Fig. 3D). Compared to the Sham group, caspase-3 expression was significantly increased in the I/R group $(1.32 \pm 0.12 ; \mathrm{p}<0.05)$. Compared to the I/R group, caspase- 3 expression was decreased in the FH group $(0.97 \pm$ $\pm 0.06 ; \mathrm{p}<0.05)$. No significant difference was found between the $\mathrm{I} / \mathrm{R}$ group and the $\mathrm{FH}+\mathrm{I}$ group $(1.42 \pm 0.11 ; \mathrm{p}>0.05)$.

7. Akt expression in myocardial tissues was not influenced by FH (Fig. 3E). There were no significant differences in Akt expression between the groups.

8. $\mathrm{FH}$ promoted the expression of P-Akt in the acute myocardial ischemia-reperfusion model (Fig. 3F). Compared to the Sham group, no significant differences in P-Akt expression were detected between the $\mathrm{I} / \mathrm{R}$ group $(1.09 \pm 0.06)$, and the $\mathrm{FH}+\mathrm{I}$ group $(1.21 \pm 0.06 ; \mathrm{p}>0.05)$, while P-Akt expression increased significantly in the FH group $(1.73 \pm 0.05 ; \mathrm{p}<0.05)$.

\section{Discussion}

Ischemia-reperfusion injury is a major obstacle to the therapy of AMI. The ability to reduce ischemia-reperfusion injury represents a clinical challenge [4]. PPost has gradually become an important way of alleviating MI/RI, replacing IPC and IPost with their limited clinical value and numerous complications.

FH is an effective drug to reduce MI/RI. In this study, we detected that FH significantly narrowed the myocardial infarct size when ischemic myocardium of rats received reperfusion (Fig. 1). The result was consistent with that of previous studies concerning fasudil post-conditioning [21], verifying that $\mathrm{FH}$ preserved post-conditioning against myocardial infarction.

Myocardial ischemia and reperfusion is a complicated pathological process [23-25]. Although $\mathrm{FH}$ has been proved to be able to activate m-KATP channels to induce PPost [21], many other mechanisms are still valuable. As the inhibitor of ROCK, $\mathrm{FH}$ plays a heart protection role via inhibiting the activity of ROCK by competing for the ATP binding site in the ROCK catalytic domain [20]. ROCK, which is also known as Rho kinase and Rho-associated kinase, is the most extensively studied downstream target of Rho [26]. Rho/ROCK is widely distributed in mammalian tissues and cells, and performs molecular switch and signal transduction functions. Hamid et al. [18] showed that ROCK was activated during early reperfusion. Inhibition of ROCK at reperfusion onset limited infarct size through an Akt/eNOS-dependent mechanism, suggesting that ROCK action at reperfusion may be deleterious through suppression of the RISK pathway [18].

In our study, we found that ROCK expression was upregulated in the acute myocardial ischemiareperfusion models, although it was not influenced by FH (Fig. 3A). So we speculated that FH PPost was solely associated with the activity of ROCK, not the expression.

Reperfusion injury salvage kinase (RISK) is a protein kinase involved in the maintenance of cell survival. In the early stages of myocardial reperfusion, RISK is activated by a kinase cascade and plays an important role in myocardial protection by inhibiting apoptosis [27]. Apoptosis is an important mechanism for MI/RI [23-25]. So we studied the relationship between $\mathrm{FH}$ and apoptosis. We found that $\mathrm{FH}$ significantly reduced cell apoptosis in ischemia-reperfusion myocardium (Fig. 2). Bcl-2 expression was increased (Fig. 3B), while Bax and Caspase-3 expression was decreased (Fig. 3C, D). However, these were abrogated following treatment with the PI3K inhibitor LY-294002 (Fig. 2, 3B-D), demonstrating that FH PPost was associated with the activation of PI3K-Akt. PI3K-Akt is an important part of the RISK signaling pathway, playing a critical role in heart protection induced by 
ROCK inhibition [18]. Our study showed that the expression of Akt in ischemia-reperfusion myocardium was not influenced by FH (Fig. 3E), but the phosphorylation of Akt was promoted by it (Fig. 3F). This indicated that $\mathrm{FH}$ was able to inhibit cell apoptosis and protect myocardium against MI/RI via the activation of PI3K-Akt. And it was thought to be associated with the suppression of the activity of ROCK.

$\mathrm{FH}$ is a novel calcium antagonistic vasodilator marketed by Asahi Chemical Company in 1994 under the trade name Erill. Currently, it is mainly used as a vasodilator to treat cerebral vasospasm induced by subarachnoid hemorrhage, pulmonary hypertension, and cognition and memory dysfunction in Alzheimer's disease.

In this study, we have confirmed the post-conditioning effect of $\mathrm{FH}$ and found it was possibly due to the reduced incidence of apoptosis following reperfusion of myocardiocytes. And the specific molecular mechanism may involve activation of PI3K-Akt signaling pathway. Therefore, $\mathrm{FH}$ may provide a novel agent for the protection of myocardium in myocardial ischemia-reperfusion.

\section{Conclusions}

FH post-conditioning was able to alleviate MI/RI, with narrowing of the infarct size and decreased apoptosis of ischemic cardiocytes. The mechanism was associated with activation of the PI3K-Akt signaling pathway.

\section{Acknowledgements}

We gratefully acknowledge the School Fund of Shanghai Jiaotong University School of Medicine (YZ1028, to Dr. Zhang) and the Project of Shanghai Baoshan Science and Technology Committee (No.10-E-4, to Dr. Zhang).

\section{Conflict of interest: none declared}

\section{References}

1. Ting P, Chua TS, Wong A, Sim LL, Tan VW, Koh TH. Trends in mortality from acute myocardial infarction in the coronary care unit. Ann Acad Med Singapore, 2007; 36: 974-979.

2. Kones R. Primary prevention of coronary heart disease: integration of new data, evolving views, revised goals, and role of rosuvastatin in management. A comprehensive survey. Drug Des Devel Ther, 2011; 5: 325-380.

3. Rosamond W, Flegal K, Furie K et al. Heart disease and stroke statistic-2008 update: A report from the American Heart Association Statistics Committee and Stroke Subcommittee. Circulation, 2008; 117: e25-e146.

4. Sanz-Rosa D, García-Prieto J, Ibanez B. The future: Therapy of myocardial protection. Ann NY Acad Sci, 2012; 1254: 90-98.
5. Murry CE, Jennings RB, Reimer KA. Preconditioning with ischemia: A delay of lethal cell injury in ischemic myocardium. Circulation, 1986; 74: 1124-1136.

6. Zhao ZQ, Corvera JS, Halkos ME et al. Inhibition of myocardial injury by ischemic postconditioning during reperfusion: Comparison with ischemic preconditioning. Am J Physiol Heart Circ Physiol, 2003; 285: H579-H588.

7. Yang XC, Liu Y, Wang LF et al. Reduction in myocardial infarct size by postconditioning in patients after percutaneous coronary intervention. J Invasive Cardiol, 2007; 19: 424-430.

8. Thibault H, Piot C, Staat P et al. Long-term benefit of postconditioning. Circulation, 2008; 117: 1037-1044.

9. Heusch G, Boengler K, Schulz R. Cardioprotection: Nitric oxide, protein kinases, and mitochondria. Circulation, 2008; 118: 1915-1919.

10. Tong $\mathrm{H}$, Chen $\mathrm{W}$, Steenbergen $\mathrm{C}$, Murphy E. Ischemic preconditioning activates phosphatidylinositol-3-kinase upstream of protein kinase C. Circ Res, 2000; 87: 309-315.

11. Tissier R, Waintraub X, Couvreur $\mathrm{N}$ et al. Pharmacological postconditioning with the phytoestrogen genistein. J Mol Cell Cardiol, 2007; 42: 79-87.

12. Del Re DP, Miyamoto S, Brown JH. RhoA/Rho kinase up-regulate Bax to activate a mitochondrial death pathway and induce cardiomyocyte apoptosis. J Biol Chem, 2007; 282: 8069-8078.

13. Yellon DM, Hausenloy DJ. Myocardial reperfusion injury. N Engl J Med, 2007; 357: 1121-1135.

14. Kuwana T, Newmeyer DD. Bcl-2-family proteins and the role of mitochondria in apoptosis. Curr Opin Cell Biol, 2003; 15: 691-699.

15. Hausenloy DJ, Yellon DM. Reperfusion injury salvage kinase signalling: Taking a RISK for cardioprotection. Heart Fail Rev, 2007; 12: 217-234.

16. Downey JM, Davis AM, Cohen MV. Signaling pathways in ischemic preconditioning. Heart Fail Rev, 2007; 12: 181-188.

17. Tissier R, Berdeaux A, Ghaleh B et al. Making the heart resistant to infarction: How can we further decrease infarct size? Front Biosci, 2008; 13: 284-301.

18. Hamid SA, Bower HS, Baxter GF. Rho kinase activation plays a major role as a mediator of irreversible injury in reperfused myocardium. AM J Physiol Heart Circ Physiol, 2007; 292: H2598-H606.

19. Wolfrum S, Dendorfer A, Rikitake Y et al. Inhibition of Rho kinase leads to rapid activation of phosphatidylinositol 3-kinase/protein kinase Akt and cardiovascular protection. Arterioscler Thromb Vasc Biol, 2004; 24: 1842-1847.

20. Jacobs M, Hayakawa K, Swenson L et al. The structure of dimeric ROCK I reveals the mechanism for ligand selectivity. J Biol Chem, 2006; $281: 260-268$.

21. Ichinomiya T, Cho S, Higashijima U, Matsumoto S, Maekawa T, Sumikawa K. High-dose fasudil preserves postconditioning against myocardial infarction under hyperglycemia in rats: Role of mitochondrial KATP channels. Cardiovasc Diabetol, 2012; 11: 28.

22. Zhang YM, Wang Y, Liu XH, Zhang DW. Cardioprotective effect of edaravone pharmacological postconditioning on acute myocardial ischemia/reperfusion injury: Experiment with rats. Zhonghua Yi Xue Za Zhi, 2008; 88: 2558-2561.

23. Gottlieb RA, Burleson KO, Kloner RA, Babior BM, Engler RL. Reperfusion injury induces apoptosis in rabbit cardiomyocytes. J Clin Invest, 1994; 94: 1621-1628.

24. Fliss H, Gattinger D. Apotosis in ischemic and reperfused rat myocardium. Circ Res, 1996; 79: 949-956.

25. Beresewicz A, Czarnowska E, Maczewski M. Ischemic preconditioning and superoxide dismutase protect against endothelial dysfunction and endothelium glycocalyx disruption in the postischemic guinea-pig hearts. Mol Cell Biochem, 1998; 186: 87-97.

26. Matsui T, Amano M, Yamamoto T et al. Rho-associated kinase, a novel serine/threonine kinase, as a putative target for small GTP binding protein Rho. EMBO J, 1996; 15: 2208-2216.

27. Hausenloy DJ, Maddock HL, Baxter GF, Yellon DM. Inhibiting mitochondrial permeability transition pore opening: A new paradigm for myocardial preconditioning? Cardiovasc Res, 2002; 55: 534-543. 